\title{
Study of Negative Electron Affinity Property for Varied Doping GaN Photocathode
}

\author{
Jianliang QIAO ${ }^{1, \mathrm{a}}$, Dayong $\mathrm{HUANG}^{1}$ and Youtang $\mathrm{GAO}^{1}$ \\ ${ }^{1}$ School of Electronic and Electrical Engineering, Nanyang Institute of Technology, NanYang \\ 473004 China \\ aqsyxn@163.com ${ }^{1}$
}

\begin{abstract}
Negative electron affinity (NEA) property means that the vacuum level is lower than the conduction band minimum in the bulk. For GaN photocathode, the effective electron affinity of the material is smaller than zero. According to Spicer "3-step model", the photoemission mechanism for varied $\mathrm{GaN}$ photocathode was studied. For the varied doping GaN photocathode, the photoelectrons can be transported to the surface by diffusing and drifting in the directional inside electric field, and the higher photoemission performance can be obtained. By covering with low escape power materials such as $\mathrm{Cs}$ or $\mathrm{O}$ on an atomically clean surface, Negative electron affinity property can be achieved for the varied doping GaN photocathode. Using the activation and evaluation system for NEA photocathode, the varied doping $\mathrm{GaN}$ photocathode was activated with $\mathrm{Cs}$ and $\mathrm{O}$, and the photocurrent curve for varied doping $\mathrm{GaN}$ photocathode was gotten.
\end{abstract}

\section{Introduction}

In recent years, with the development of information and detection technology, UV detection technology become an important optoelectronic detection technology that used in both military and civilian. As a key part of UV vacuum detect device, UV photocathode decides the whole performance of detect devices. Negative electron affinity GaN photocathode is an ideal new kind of UV photocathode. It has many virtues, such as high quantum efficiency, low dark current, concentrated electrons energy distribution and angle distribution, adjustive threshold and so on ${ }^{[1,2]}$.

Stanford University has done much work about the preparation of NEA GaN photocathode and surface mechanism ${ }^{[3,4]}$. NASA Goddard Space Flight Center have gotten the important results from Cs activated GaN photocathode development for MCP detector systems ${ }^{[5]}$. Northwest University studied the fabrication and the development of GaN photocathode for imaging detectors ${ }^{[6,7]}$. Hamamatsu Corporation have already succeeded in preparing NEA GaN photocathode ${ }^{[8]}$.

But the photoemission mechanism and preparation technique for NEA GaN photocathode, especially to varied doped $\mathrm{GaN}$ photocathode are still to be researched further. Encircling NEA GaN photocathode photoemission mechanism and the activation technics, using the NEA photocathode multi-information test and evaluation system,

* Corresponding author: qsyxn@163.com 
negative electron affinity property for varied doping GaN photocathode was studied in the paper.

\section{Experimental Setup}

The photograph of NEA photocathode activation and evaluation experiment system is shown in Fig. 1. the whole system includes five parts: the ultra-high vacuum (UHV) activation system, the multi-information measurement system, the surface analysis system,the X-ray photoelectron spectroscopy (XPS), the ultraviolet ray photoelectron spectroscopy (UPS) and the quadrupole mass-spectrometer. As an intelligent and multifunctional system, the whole system has many advantages such as powerful functions, high degree of automation, rich test object and test precision higher etc.. The photocathode activation, surface analysis and spectral response mesurement can be done in this system.

The ultra-high vacuum activation system is used to clean and activate photocathode. The surface analysis system is composed of the X-ray photoelectron spectroscopy and the ultraviolet ray photoelectron spectroscopy, can test the information of sample surface on line. The UHV activation system and the surface analysis system are connected with an insulating valve. The samples can be easily moved between the UHV activation chamber and the surface analysis room by a magnetic transfer pole. The multi-information measurement system can measure the photocurrent, the Cs source current, the $\mathrm{O}$ source current, the vacuum degree and the spectral response etc.. And the mass spectrometer can be used to analysize the residual gas in the ultra high vacuum system. The diagram of NEA photocathode activation and evaluation experiment system is shown in Fig. $2^{[9,10]}$.

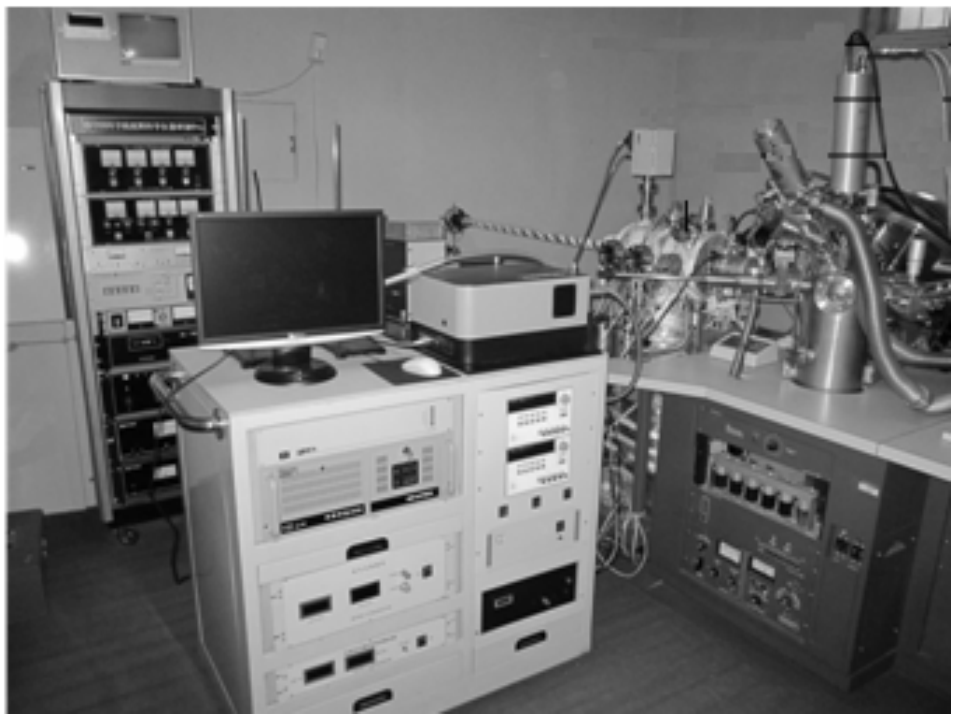

Fig. 1 The photograph of NEA photocathode activation and evaluation experiment system 


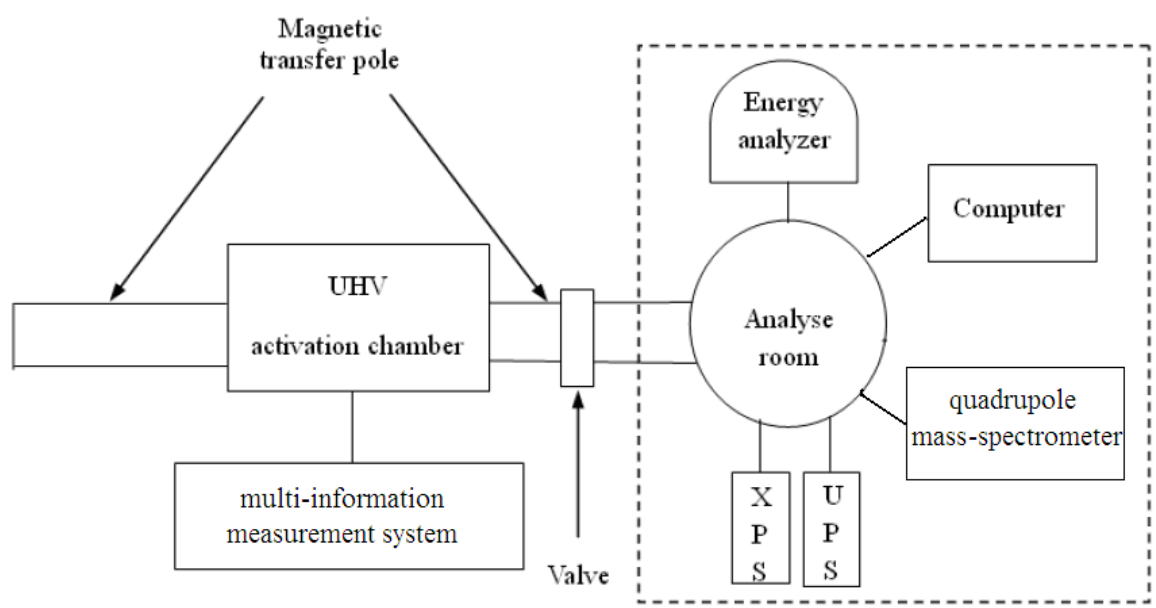

Fig.2 The diagram of NEA photocathode activation and evaluation experiment system

\section{Photoemission Mechanism for NEA GaN Photocathode}

The structure of NEA GaN photocathode can be divided into the substrate,the buffer layer and the activation layer. The photoemission mechanism of NEA GaN photocathode can be explained with Spicer photoemission "3-step model": light absorption, photoelectron transmission and photoelectron emission ${ }^{[11]}$. NEA GaN photocathode is sensitive to the UV light under $365 \mathrm{~nm}$. The photoelectrons escaping from the UV photocathode can be emitted to the free space. The " 3 -step model" photoemission course is shown as Fig. $3^{[2]}$.

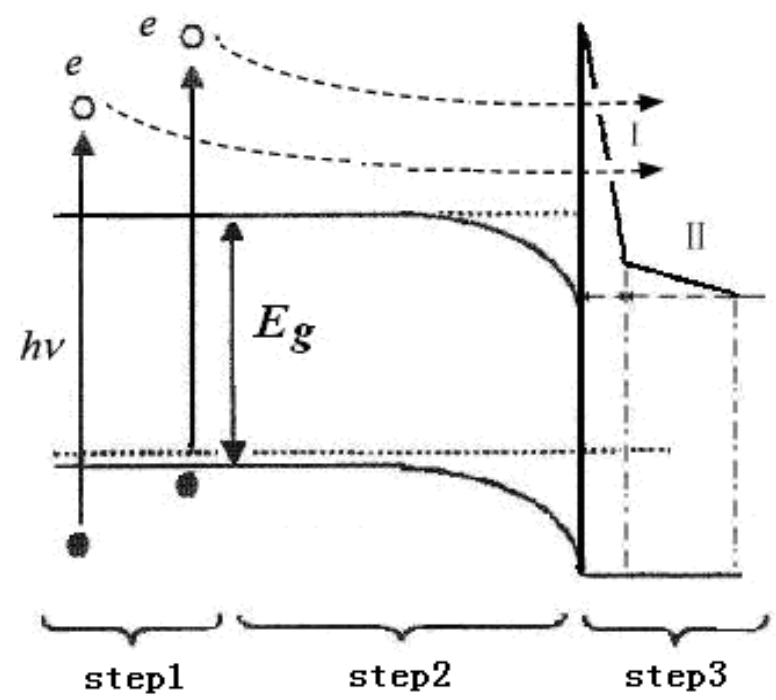

Fig.3 The photoemission process of NEA GaN photocathode

The first step is the absorption of incidence UV light. Under the irradiation of uv light, the photon energy is absorbed by the electrons in the valence band. The electrons with enough energy in the valence band are stimulated to the conduction band. The main source 
of photoemission for NEA GaN photocathode is the escape of hot electrons. The first step is decided by the photon energy and the material band structure.

The second step of " 3 -step model" is the transportation of photoelectron. To the uniform doping material, the excitated photoelectrons in the conduction band will move from the bulk to the surface by diffusion movement. To the varied doping material, because of the directional inside electric field, the photoelectrons can be transported to the surface by diffusing and drifting. This transportation of photoelectron includes the bulk transportation and the surface band bend transportation. The lifetime of photoelectron is also an important factor to the whole transportation.

The third step is that the photoelectrons pass through the surface potential and escape to the vacuum. Because of NEA characteristic, the photoelectrons arriving at the surface can pass through the surface potential and escape into the vacuum. The energy band will bend downwards properly by p-type doped process. The width of barrier layer will be as narrow as possible with heavily doped method in order to make the ionization impurity move to the minimum distance.

\section{Activation of the Varied Doping NEA GaN Photocathode}

The p-type varied doping GaN sample was grown by metal organic chemistry vapour phase deposits technology. The buffer layer is AIN, the substrate is c-axis sapphire. and the doping element is $\mathrm{Mg}$. The sample active layer thickness is about $185 \mathrm{~nm}$. The doping concentration of active layer is respectively about $1.0 \times 10^{18} \mathrm{~cm}^{-3}, 4.0 \times 10^{17} \mathrm{~cm}^{-3}, 2.0 \times 10^{17}$ $\mathrm{cm}^{-3}$ and $6.0 \times 10^{16} \mathrm{~cm}^{-3}$ from bulk to surface.

In order to remove the carbon contamination from surface, the GaN material was degreased and chemically cleaned. After the chemical cleaning, the heat cleaning was required to get the atom clean surface in the NEA photocathode activation and evaluation experiment system. The carbide and oxid on GaN surface were decomposed by about 20 minutes heat cleaning.

With continuous Cs flux and interrupted $\mathrm{O}$ flux, the varied doping GaN photocathode sample was activated in UHV activation chamber. The curve of photocurrent during $\mathrm{Cs} / \mathrm{O}$ activation for varied doping GaN photocathode is shown in Fig. 5. During Cs/O activation process, the Cs flux was always on, and the $\mathrm{O}$ flux was on or off alternately according to the photocurrent value.

By covering low escape power materials such as $\mathrm{Cs}$ and $\mathrm{O}$ alternately on atomical clean surface of $\mathrm{GaN}$ photocathode, the negative electron affinity state was achieved. The varied doping GaN photocathode was activated successfully. Fig. 4 is the curve of photocurrent during $\mathrm{Cs} / \mathrm{O}$ activation for varied doping $\mathrm{GaN}$ photocathode ${ }^{[12]}$. 


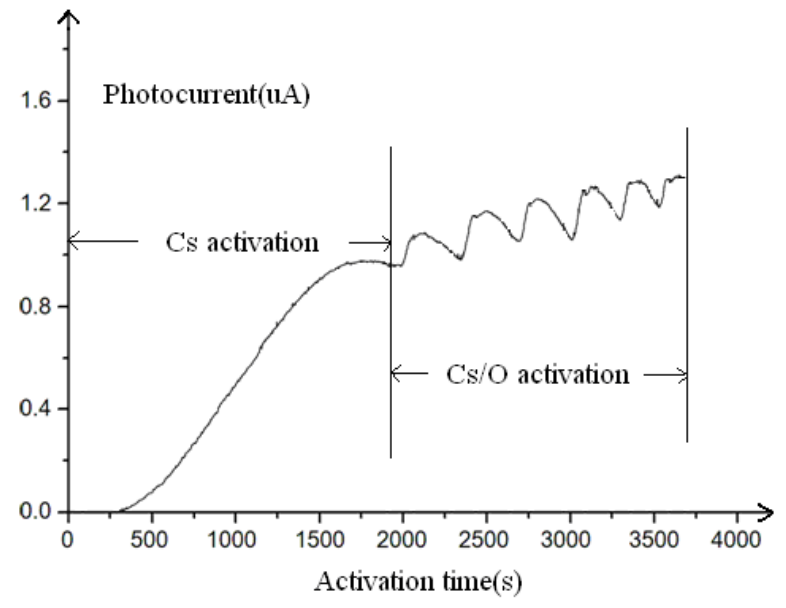

Fig. 4 The curve of photocurrent during $\mathrm{Cs} / \mathrm{O}$ activation for varied doping $\mathrm{GaN}$ photocathode

\section{Conclusion}

As an ideal new kind of UV photocathode with many virtues, Negative electron affinity GaN photocathode will acquire wide applications in UV detection and vacuum electron source. According to the theory of Spicer "3-step model": light absorption, photoelectron transmission and photoelectron emission, negative electron affinity property for $\mathrm{GaN}$ photocathode was studied. The energy of photon absorbed in the valence band decides if the hot electrons can be produced in the conduction band. To the varied doping material, the factors affecting the transportation of photoelectrons include the diffusion and the drifting. So the higher photoemission performance can be obtained. For NEA GaN photocathode, the photoelectrons arriving at the surface can easily pass through the surface potential. With continuous $\mathrm{Cs}$ flux and interrupted $\mathrm{O}$ flux, the varied doping $\mathrm{GaN}$ photocathode sample was activated using the activation and evaluation system for NEA photocathode.

\section{Acknowledgment}

This work was supported by National Natural Science Foundation of China (Grant No. 61371058).

\section{References}

1. O.H.W. Siegmund, A. S. Tremsin, J. V. Vallerga, J. B. McPhate, J. S. Hull, J. Malloy and A. M. Dabiran, Gallium nitride photocathode develop.ment for imaging detectors, Proc. of SPIE, 2008, 7021:70211B.

2. Wang Xiao-Hui, Shi Feng, Guo Hui, Hu Cang-Lu, Cheng Hong-Chang, Chang Ben-Kang, Ren Ling, Du Yu-Jie,Zhang Jun-Ju. "The optimal thickness of a transmission-mode GaN photocathode", Chinese Physics B, 2012,21(8), 087901.

3. Machuca F, Liu Z, Sun Y, et al. Role of oxygen in semiconductor negative electron affinity photocathodes. J. Vac. Sci. Technol.B. 2003, Vol. 20, No. 6, 2721-2725. 
4. Machuca F, Liu Z, Sun Y, et al. Oxygen species in $\mathrm{Cs} / \mathrm{O}$ activated gallium nitride (GaN) negative electron affinity photocathodes. J. Vac. Sci. Technol. 2003, 21(4), 1863-1869.

5. Timothy Norton, Bruce Woodgate, Joseph Stock, George Hilton, Mel Ulmer, Shahid Aslam, R.D Vispute. Results from Cs activated GaN photocathode development for MCP detector systems at NASA GSFC. Proceedings of SPIE, 2003, 5164: 155-164.

6. M. P. Ulmer, W. B. Wessels, F. Shahedipour, R. Y. Korotkov, C. Joseph, and T. Nihashi. Progress in the fabrication of GaN photo-cathodes. Proceedings of SPIE,2001, Vol. 4288,246-253.

7. M. P. Ulmer, B. W. Wessels, and O. H. W. Siegmund, Advances in wide-band gap semi-conductor based photocathode devices for low light level applications, Proc. of SPIE, 2002, 4650,94-103.

8. Shahedipour F S, Ulmer M P, et al. Efficient GaN photocathodes for low-level ultraviolet signal detection[J]. IEEE Journal of Quantum Electronics, 2002, 38(4): 333-335.

9. J.J. Zou, Y.S Qian, B.K. Chang, G.Y. Lin, L. Feng, Design of multi-information measurement system for GaAs photocathodes[J], Semiconductor Optoelectronics(China), 2006, 27(5): 582-585.

10. J.J. Zou, Y.S. Qian, B.K Chang, H. Wang, S.Y. Wang, Multi-information measurement technology for GaAs photocathode preparation[J], Journal of Vacuum Science and Technology(China), 2006, 26(3):172-175.

11. W.E.Spicer and A.Herrera-Gómez. Modern theory and application of photocathodes. Proc. SPIE, 1993, 2022: 18-33.

12. Wang Xiao-Hui, Photoemission performance of wurtzite structure GaN (0001) surface [D], Nanjing University of Science \& Technology, 2013,75. 